\title{
Effects of age and asymmetrical stimulation on the subjective median plane'
}

\author{
ROBERT H. POLLACK ${ }^{2}$ AND DOROTHY JEAN CARTER \\ INSTITUTE FOR JUVENILE RESEARCH
}

\begin{abstract}
Changes in subjective median plane (SMP) as a result of asymmetrical visual and bodily stimulation were measured for a total of 260 Ss, age 5-17. Asymmetrical visual stimulation resulted in a readjustment of the SMP in the direction of stimulation, with a decline in magnitude of response as a function of age. Asymmetrical bodily stimulation was assimilated to by the younger children (SMP adjusted in the direction of stimulation), and counteracted by the older children (age 8 and older). In the combination of the two, the effects of visual stimulation dominated those of bodily stimulation throughout the age range, possibly due to insufficient exposure to the asymmetrical bodily stimulation. Correlational analysis showed that $C A$, rather than $M A$, was the major variable associated with the results. The results are in general agreement with sensory-tonic theory, and are discussed in this light.
\end{abstract}

Sensory-tonic theory postulates that "in dealing with perception one must consider the relationship between object-stimuli (psychophysical) and organismic state (psychophysiological)." (Wapner \& Werner, 1957, p. 1.) Underlying this basic relationship is the concept of stability; i.e., a fundamental biological tendency of the organism is toward the stabilization of this object: organism relationship. Thus, if any unchanging stimulus is in an unstable (asymmetrical) relationship to the ongoing state of the organism, the tendency is for the organism to change its state so that a stable relationship results.

There are, however, ontogenetic changes in this relationship: "... development proceeds in terms of an increase in differentiation and hierarchic integration." (Wapner \& Werner, 1957, p. 12). At early ages, there is a relative lack of differentiation of self and object, due to the effects of (1) "egocentricity"; i.e., determination of the objective world through the body as a frame of reference, and (2) of "stimulus boundedness"; i.e., the dominating effect of the initially-presented stimulus. As the organism in eneral matures, and increasing differentiation and hierarchical organization takes place, self and object are increasingly differentiated, resulting in a more objectified external space.

Stimuli can, of course, impinge upon the organism not only from external objects, but from within the organism itself. In sensory-tonic terminology, any stimulation emanating from the object immediately attended to is called object stimulation, and any other stimulation occurring at the same time-bodily, that from another object-or that occurring independent of an object, is called extraneous. Asymmetrical extraneous stimulation produced, for example, by holding one arm to the side or tilting the head to one side, can be responded to in either of two ways. The observer can assimilate to it, adjusting his perceptual dimensions toward the source of stimulation, or he can counteract it, compensating for this stimulation by adjusting his perceptual dimensions in the opposite direction. The response to asymmetrical object stimulation produced, for example, by the extension of an object to either side of the center of the visual field, should be in the direction of stimulation, but this effect should decrease with age.

Various investigations have supported the theoretical and developmental implications of sensory-tonic theory. In an experiment concerning the effect of fixation on the perceived distance of a three-dimensional rod, Pollack (1967) found that younger children assimilated to the extraneous bodily stimulation (the strain of convergence) by moving the non-fixated rod toward themselves after the period of fixation, indicating that they had perceived the fixated rod as having moved toward themselves during the fixation period. Older children, however, counteracted the extraneous stimulation and moved the non-fixated rod away from themselves. This assimilation-counteraction shift occurred at about age seven. Wapner and Werner, in a study of the effect of body tilt on the perception of the vertical (1957), also found that younger children assimilated to the extraneous stimulation, by adjusting a visible vertical rod in a direction identical to that of the body tilt, while the older children adjusted the vertical in the opposite direction. In this study, however, the assimilationcounteraction shift occurred early in adolescence.

In addition, Pollack (1961) has found, in an experiment involving shifts in the apparent median (saggital) plane produced by asymmetrical stimulation and fixation, that when asymmetrical object and extraneous stimulation occur at the same time, they appear to be of equal potency for adults.

Thus the present study was conducted over a large range of ages, in order to determine the effects, both singly and in interaction, of asymmetrical object and extraneous (bodily) stimulation on the subjective median plane. It was expected that asymmetrical visual stimulation would produce responses in the same direction as the stimulation occurred, due to the effects of "stimulus boundedness," but that this effect would diminish with age, reflecting increasing differentiation in children at increasingly older age levels. On the basis of the studies mentioned above, it was expected that, for young children, asymmetrical bodily stimulation would lead to assimilation of the stimulation, with a shift to counteraction somewhere within the tested age range. Finally, it was expected that the oldest children would respond as adults, showing the equal effectiveness of the two types of stimulation in their interaction.

\section{SUBJECTS $^{3}$}

Ss were 10 boys and 10 girls in each grade from kindergarten through the senior year of high school, yielding a chronological age range of from 5 years 7.5 months to 17 years 5 months. The children all attended suburban schools and had 20/20 uncorrected vision.

\section{APPARATUS}

All testing was carried out in a mobile research laboratory, painted flat black inside, which was leveled in place. The apparatus used was the Apparent Eye-line and the Median Plane Apparatus, Model V-0162, manufactured by the Polymetric Company of Reading, $\mathrm{Pa}$. Its luminous square was viewed at $5 \mathrm{~m}$ by the $\mathrm{Ss}$, and subtended a visual angle of $2 \mathrm{deg} 12 \mathrm{~min}$ at this distance. Brightness of the square was kept constant at $.46 \mathrm{ft}-\mathrm{c}$, and its speed of movement at $2.3 \mathrm{~cm} / \mathrm{sec}$. Whenever the middle of the square was under consideration, a Bandaid plastic "dot" was placed in the middle of the square. A numeral "1" was taped at the left edge of the square, and a numeral " 2 " at the right edge. A dry sponge taped to the wall behind the experimental chair provided $S$ with a head rest.

\section{PROCEDURE}

For each session, the child was brought into the experimental chamber and placed in the experimental chair. Procedure was explained with the aid of a cardboard demonstrator. $S$ was told he would be asked to set either the center of the square, or one of its edges, "straight ahead," "in front of your nose." He was told of the importance of keeping his body straight in the chair, and of keeping his head still. A series of practice trials was carried out with the cardboard square. S told $\mathrm{E}$ to move the square toward the 
Table 1

Experimental Design

\begin{tabular}{lccc}
\hline & \multicolumn{3}{c}{ Visual Stimulation } \\
Bodily Stimulation & Left Sq Extent & Right Sq Extent & No Sq Extent \\
\hline Left Arm & $X$ & $X$ & X \\
Right Arm & X & X & X \\
No Arm & $X$ & $X$ & X(Base SMP)
\end{tabular}

" 1 " side or toward the " 2 " side to line it up, and to say "stop" when the middle, or edge, of the square appeared to be straight ahead. S was then dark-adapted for $5 \mathrm{~min}$, and the session was begun.

With the square at objective " 0 " each time, eight control settings were taken, for which $S$ lined up the center of the square. Objective "O" for S was found by lining up the middle of the chair in which $S$ sat to coincide with objective " 0 " on the apparatus. As indicated above, $S$ instructed $E$ which way to move the square each time, and thus exercised no direct control over its movement. The square moved at a constant speed, and once $S$ had said "stop," no fine adjustments were permitted. Between trials throughout, $S$ was prohibited from viewing the square while $E$ made recordings and readjustments, by a second $\mathbf{E}$ who drew a screen in front of $S$. The average of the eight control settings was taken as S's subjective median plane (SMP), and subsequent measurements were deviations from this measure.

The conditions employed are shown in Table 1. Both extent conditions were presented, to control for position effects. Square extent was given by placing the right (for left extent) or left (for right extent) edge of the square at S's SMP. Arm extent was given by $\mathrm{S}^{\prime}$ holding one arm laterally at shoulder height for $15 \mathrm{sec}$ while looking at the square, whose center was set at the SMP. S then had to readjust the square so that the center appeared to be straight ahead. In addition to the four conditions presented in Session 1, the four interaction conditions outlined above were presented in Session 2, held several days later.

Session 1 involved the arm and square extent conditions, each presented first to a different half of the Ss, with eight trials for each arm or extent, counterbalanced for arms or edges. Session 2 involved the four interaction conditions, each presented eight times and counterbalanced for arms and edges. $A$ rest was given in each session at the halfway point. For each of the eight conditions from the two sessions, the average of the eight trials was taken as the score for that $S$.

\section{RESULTS}

Since an initial examination of the data revealed no sex differences, boys' and girls' scores were combined. Movement toward the direction of square extent was arbitrarily coded as (t), and movement in the opposite direction as (-). There were four

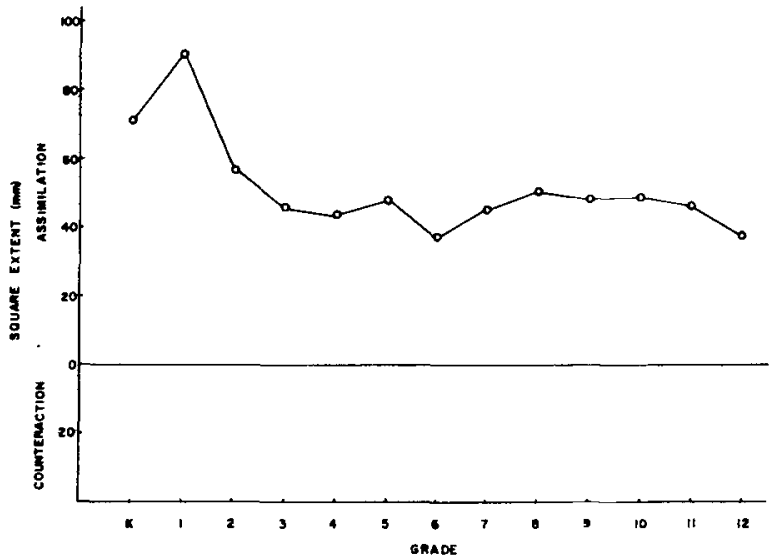

F. 1: Sum of left and right aquare extent acorea. scores for each S: (1) sum of left and right square extent scores, (2) sum of left and right arm extent scores, (3) sum of extent: like-arm-raised scores (e.g., left extent-left arm, and right extentright arm), and (4) sum of extent: unlike-arm-raised scores.

Figures 1 and 2 present the square extent and arm extent scores. Analysis of variance performed on the square extent scores revealed a significant declining age trend $(F=3.14$, $\mathrm{df}=12 / 247$, $p<.01$ ). Analysis of variance performed on the arm extent scores also showed a significant age effect $(F=2.01$, df $=12 / 247$, $\mathrm{p}<.01$ ). This change, as can be seen in the graph, is produced by a shift from assimilation of the effects of arm extension to counteraction of them; i.e., the younger children adjusted the square to the left with the left arm extended, and to the right with the right arm extended, while the older children adjusted the square in the direction opposite that of the extended arm.

Figure 3 presents the graph for the conditions combining square and arm extent. Analysis of variance revealed significant effects of age $(F=5.84, \mathrm{df}=12 / 494, \mathrm{p}<.01)$ and condition $(F=4.67$, $\mathrm{df}=1 / 494, p<.01)$ but no significant interaction $(F=.30$, $\mathrm{df}=12 / 494)$. Inspection of the graph shows that the age effect is due to an overall decrease in magnitude of response, and that the condition effect is due to a consistently greater response in the direction of square extension when the arm opposite that direction is raised. This contribution of arm extension to the response evoked by object stimulation, however, is relatively small.

Table 2 presents the results of a correlational analysis of the data. The values include only Grades 1-12; the kindergarten children had to be omitted in the analysis, due to lack of MA scores. The score used for analysis of the interaction conditions was simply Score (4) above minus Score (3) for each S. From the table it can be seen that the major variable associated with the arm extent scores is CA. It is significantly and positively related to the scores, while MA is not; furthermore, partial correlation, holding MA constant, elevates the degree of relationship. MA is significantly and negatively correlated with the square extent scores, while the vahue of $r$ for $C A$ does not reach significance, but partial correlation, holding $C A$ constant, lowers the MA-extent $r$ value. It can be seen from the partial $r$ values that $M A$ and $C A$ are correlated with the various scores in opposite directions, and thus are working against each other. In view of the magnitude of the MA-interaction partial $r$, it is surprising that the CA-interaction $r$ reaches the level of significance that it does. Thus, considering all the correlations, it seems justified to conclude that chronological age, rather than intellectual functioning, plays the predominant role in the results encountered here.

\section{DISCUSSION}

The results are in general agreement with sensory-tonic theory. Asymmetrical object stimulation produced a shift in the SMP in the direction of stimulation for all Ss. However, this effect

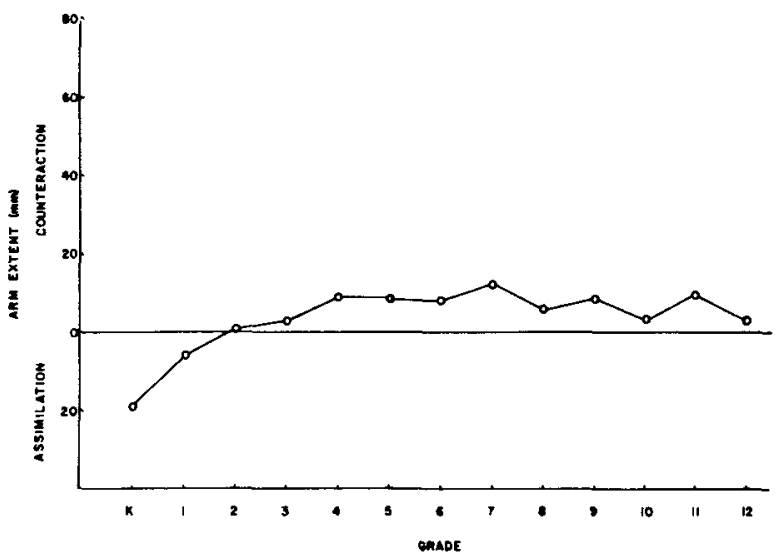

Fi. 2. Sam of left and right arm extent roores. 


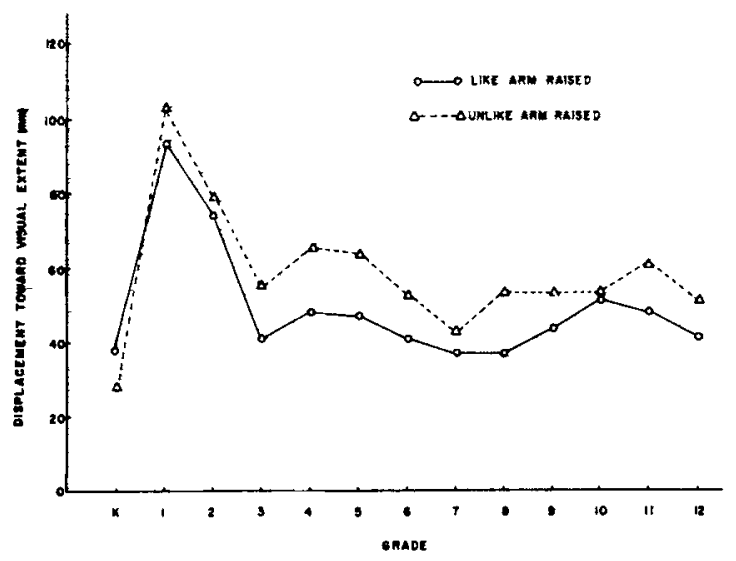

Fie. 3. Interaction scores: Displincement toward visual extent with like arm rained, and with unlike arm raived.

diminished with age, indicating that the degree of "stimulus boundedness" decreases, which is to be expected as a concomitant of increasing differentiation of self and object on the part of the older children. Asymmetrical extraneous stimulation produced a shift in the SMP in the direction of stimulation for the six- and seven-year olds (assimilation), and in the opposite direction for the older children (counteraction). This assimilation-counteraction shift thus again occurred at about age seven, as in Pollack's study with the three-dimensional vertical rod. Another study pointing to age seven as a critical one for certain perceptual experiences is that of Wohlwill (1963), concerning the perception of size and distance as a function of chronological age. He found that at age seven, a shift from underconstancy to overconstancy occurs, possibly resulting from a shift from assimilation to convergence to counteraction of it. Our results suggest that this shift occurs relatively independent of intellectual functioning, and an earlier study by Jenkin and Feallock (1960) would seem to lend support to this. In their study, the relationship between size-distance judgments and intelligence was investigated, and when samples of normals and retardates were matched for $\mathrm{CA}$, no differences were found. All of these findings suggest that the assimilationcounteraction shift is a fairly low-level cognitive one, involving a change in the mode of responding to muscular stimulation, and that once the change is made, no further development occurs.

The surprising thing in the present study is that even at age 17, the interaction scores show that visual stimulation continues to dominate the effects of muscular involvement-"stimulus boundedness," though diminished in degree of influence, is still very much in operation. If, as in Pollack's (1961) study with adults, the two sources of stimulation were of equal potency, wherever they were opposed the resultant response should reflect a cancellation effect. Thus, in the present study, scores for left extent-left arm and right extent-right arm should be about zero. This, however, is not the case. It is very possible that the $15 \mathrm{sec}$ allowed for arm extension was an insufficient amount of time for the muscular
Table 2

Correlations Between MA, CA, and SMP Displecement Scores

\begin{tabular}{lcc}
\hline Variables & $\mathrm{r}$ & $\mathrm{p}$ level \\
\hline CA-square extent & -.1196 & NS \\
CA-arm extent & .1294 & .05 \\
CA-interaction & .1896 & .01 \\
MA-square extent & -.1573 & .01 \\
MA-arm extent & .0878 & NS \\
MA-interaction & .044 & NS \\
MA-CA & .9372 & .001 \\
& & \\
CA-square extent, MA constant & & \\
MA-square extent, CA constant & .0807 & NS \\
CA-arm extent, MA constant & -.1308 & .01 \\
MA-arm extent, CA constant & .1358 & .01 \\
CA-interaction, MA constant & .0965 & NS \\
MA-interaction, CA constant & .4299 & .001 \\
\hline
\end{tabular}

effects to exercise more than a minimal influence. $A$ fact lending support to this conclusion is that in Pollack's study with adults, the extended arm was held at shoulder height for $2 \mathrm{~min}$, affording ample time for muscular effects to come into play. In the present study, $15 \mathrm{sec}$ for arm extension was chosen, for fear that any longer period would be difficult to maintain with the younger children. Considering the overall agreement of the results of this study with those of previous studies, and with the hypothesis formulated, it seems likely that a longer period of arm extension for the children might have yielded the expected results.

\section{REFERENCES}

JENKIN, N., \& FEALLOCK, S. M. Developmental and intellectual processes in size-distance judgment. Amer. J. PsychoL, 1960, 73, 268-273.

POLLACK, R. H. Apparent median plane shifts with asymmetrical stimulation and fixation. Austral. J. Psychol, 1961, 13, 195-205.

POLLACK, R. H. Changes in the effects of fixation upon apparent distance in the third dimension. Psychon Sci, 1967, 8, 2, 83-84.

WAPNER, S., \& WERNER, H. Perceptual development. Worcester, Mass.: Clark University Press, 1957.

WOHLWILL, J. F. The development of "over constancy" in space perception. In L. P. Lipsitt \& L. L. Spiker (Eds.), Advances in child development and behavior. New York: Academic Press, 1963. Pp. 265-312.

\section{NOTES}

1. This research was supported by Grant No. HD 01433 awarded by the National Institute of Child Health and Human Development.

2. Address: Institute for Juvenile Research, 232 East Ohio St., Chicago, III. 60611.

3. The authors are grateful for the cooperation of Mr. Darrell Holsteen, Superintendent, School District 89; Dean W. Stoakes, Ph.D., Superintendent, School District 87; and principals Mr. Ronald Howard, Mr. Robert Whitaker, Mr. John Sheahan, and their staffs. In addition, we wish to acknowledge the indispensable assistance of Dr. Eugene Skoff and Mr. Richard Ptashne.

(Accepted for publication June 24, 1968) 